\title{
Orbital Evolution of the Kuiper Belt
}

\author{
Charles Morgan and Andrew Prentice
}

\section{School of Mathematical Sciences, Monash University, 3800 Australia}

\begin{abstract}
The observed distribution of trans-Neptunian objects (TNOs) implies that they originally orbited in a narrow ring of radius $41 \mathrm{AU}$. The mass of the largest TNO was around $1-4 \times 10^{26} \mathrm{~g}$.
\end{abstract}

Scattering by the largest TNO forms the stable low inclination $\left(i \lesssim 8^{\circ}\right)$ population (Fig 1a). However, most TNOs remain in the 40-42 AU unstable zone or are scattered into the chaotic fringes of the 2:3 Neptune resonance, where their inclinations and eccentricities fluctuate increasingly until they are scattered by Neptune (Fig 1b). Thus 2 distinct populations emerge from the initial ring. The 2:3 resonance is the main route to the Neptune scattered population. Stabilization of a small fraction of the TNOs passing through gives the Plutinos.

(a)

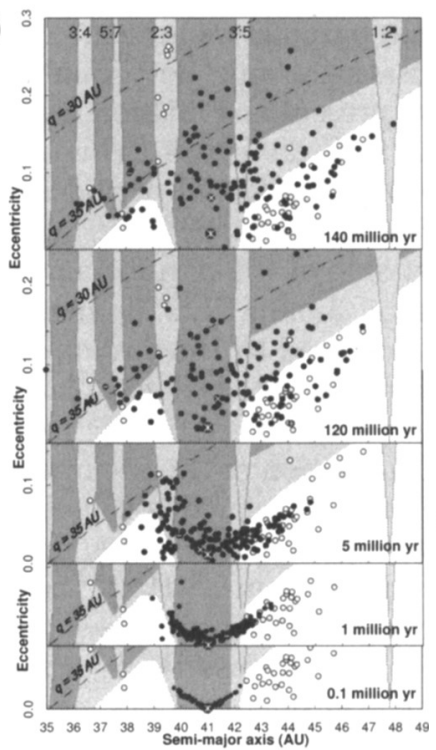

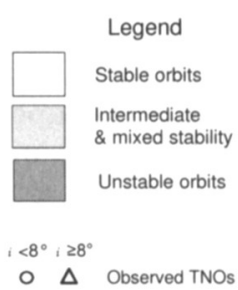

(a) Simulated low inclination TNOs

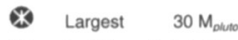

* 2nd biggest $7.1 \mathrm{M}_{\text {pluto }}$

$\oplus$ 3rd biggest $2.3 \mathrm{M}_{\text {puto }}$

- 4th biggest $0.9 \mathrm{M}_{\text {puto }}$

- Small $m \geq 0.01 \mathrm{M}_{\text {purto }}$

(b) Simulated high \& low inclination TNOs

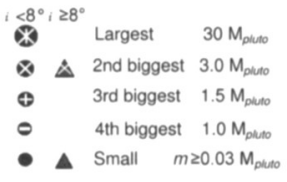

Figure 1. (a) Simulated development of the classical $\left(i \leq 8^{\circ}\right)$ population with weak planetary perturbation. The 1:2 resonance would limit expansion. (b) Real planetary perturbation gives both high and low inclination populations. The orbital stability map is adapted from Duncan, Levison, \& Budd (1995) and Malhotra (1996).

\section{References}

Duncan, M., Levison, H., \& Budd M. 1995 AJ, 110, 3073

Malhotra, R. 1996 AJ, 111, 504 